\title{
Genome-Wide Identification of Tomato Golden 2- Like Transcription Factors and Abiotic Stress Related Members Screening
}

\section{Ziyu Wang}

Northeast Agricultural University

Junfang Liu

Northeast Agricultural University

Haiyan Zhao

Northeast Agricultural University

Xuying Sun

Northeast Agricultural University

Tairu Wu

Northeast Agricultural University

Tong Pei

Northeast Agricultural University

Yue Wang

Northeast Agricultural University

Qifeng Liu

Northeast Agricultural University

Huanhuan Yang

Northeast Agricultural University

He Zhang

Northeast Agricultural University

Jingbin Jiang

Northeast Agricultural University

Jingfu Li

Northeast Agricultural University

Tingting Zhao

Northeast Agricultural University

Xiangyang Xu ( $\nabla x x y 709 @ 126 . c o m)$

Northeast Agricultural University 
Keywords: Tomato, G2-like family, Bioinformatics analysis, Expression patterns, Abiotic stresses

Posted Date: November 3rd, 2021

DOI: https://doi.org/10.21203/rs.3.rs-1007193/v1

License: (c) (1) This work is licensed under a Creative Commons Attribution 4.0 International License. Read Full License 


\section{Abstract}

Background: Golden 2-Like (G2-like) transcription factors play an important role in plant development. However, the roles of these G2-like regulatory genes in response to abiotic stresses in tomato are not well understood.

Results: In this study, we identified 66 putative G2-like genes in tomato (Solanum lycopersicum) and classified them into 5 groups (I to $\mathrm{V}$ ) according to gene structure, motif composition and phylogenetic analysis. The G2-like genes were unevenly distributed across all 12 chromosomes. There were nine pairs of duplicated gene segments and four tandem duplicated SIGlk genes. Analysis of the cis-regulatory elements (CREs) showed that the promoter regions of SIGlks contain many kinds of stress- and hormonerelated CREs. Based on RNA-seq, SIGlks were expressed in response to three abiotic stresses. Thirty-six differentially expressed S/G/ks were identified; these genes have multiple functions according to Gene Ontology (GO) analysis and are enriched mainly in the zeatin biosynthesis pathway. Further studies exhibited that silencing S/G/k16 in tomato would reduce drought stress tolerance by earlier wilted, lower superoxide dismutase (SOD), peroxidase (POD) activities, less Pro contents and more MDA contents.

Conclusion: Overall, the results of this study provide comprehensive information on G2-like transcription factors and G2-like genes that may be expressed in response to abiotic stresses.

\section{Background}

Transcription factors (TFs), also known as trans-acting factors, constitute a group of proteins that play important roles in gene regulation by activating or repressing the transcription of downstream target genes, consequently controlling many cellular activities during plant growth and development [1-3]. TFs interact with DNA promoters through DNA-binding protein domains (DBDs) [4]. Plant growth and productivity are under constant threat from environmental stimuli in the form of biotic and abiotic stresses, and TFs participate in activating or inhibiting transcription in response to these stresses [5-7]. To date, 60 different TF families have been identified in plants [8]. In this study, we investigated the Golden 2-like (also called G2-like or Glk) TF gene family in tomato.

Golden 2 (G2) was first identified in maize, and its nomenclature follows that of golden 1, the first goldenproducing factor [9]. Researchers have subsequently proved that $G 2$ acts as a novel transcriptional regulator of cellular differentiation in maize leaves [10]. GLK proteins belong to the GARP superfamily of TFs [6], which includes $\mathrm{G} 2$ in maize, Arabidopsis RESPONSE REGULATOR-B (ARR-B) proteins and the PHOSPHATE STARVATION RESPONSE1 (PSR1) protein in Chlamydomonas [11, 12]. Most G2-like genes have two highly conserved domains: a DBD (containing a helix-loop-helix [HLH] motif) and a C-terminal GCT box [13]. The DBD sequence is highly conserved among members of the GARP superfamily [14], occurring in both green algae and land plants; however, the GCT box is found only in land plants and is specific to the $G L K$ genes [15]. In many land plants, GLKs encode a pair of partially redundant nuclear 
TFs. GLK TFs are important for the expression of nuclear photosynthesis-related genes and for chloroplast development [16].

GLK genes have been identified in moss (Physcomitrella patens), Arabidopsis, rice (Oryza sativa), pepper (Capsicum annuum) and tomato to date [15,17-19], and these genes play roles in many aspects of plant biology. One vitally important function of $G L K s$ is the regulation of chloroplast development. Previous studies have shown that, compared the wild type, g2 mutants had smaller chloroplasts and reduced amounts of thylakoid lamellae [20]. GLKs are also related to cellular differentiation. Three types of chloroplasts are present in C4 plants: C4 bundle sheath (BS) cells, C4 and C3 M (mesophyll) cells [21]. In contrast, in C3 plants, two GLKs act redundantly in chloroplast development [18]. GLKs regulate chloroplast development not only in the leaves but also in the fruit; these TFs can influence fruit quality by altering the sugar and carotenoid contents, as shown by studies in Arabidopsis, pepper and tomato [17-19, 22]. In addition, overexpression of GLK TFs in Arabidopsis leads to photosynthesis in root chloroplasts [23]. GLKs also play important roles in disease defense, as has been shown mainly in Arabidopsis [24-26] in low temperature and drought stress conditions [27]; in tolerance to ozone [28]; in terms of nitrogen-use efficiency [29]; and in the regulation of leaf senescence [30]. Y Yuan, X Xu, Z Gong, Y Tang, M Wu, F Yan, X Zhang, Q Zhang, F Yang, X Hu, et al. [31] reported that overexpression of SIARF6A, which could physically bind the two TGTCTC motif in the SIGLK1 promoter, increases SIGLK1 transcriptional levels, and leads to enhanced chlorophyll accumulation and strong photosynthesis in tomato plants, which may result in strong tolerances against abiotic stresses such as drought and heat. Furthermore, GLKs are involved in the mediation of ubiquitin signaling [32].

However, previous studies of G2-like TFs in tomato have focused mainly on chloroplast development. Little is known about the roles of G2-like TFs in the stress resistance of tomato. In this study, we identified 66 G2-like genes via comprehensive bioinformatics analysis including gene structure, exon-intron organization, motif composition, gene duplication, chromosome distribution, phylogenetic relationships and promoter cis-elements. The expression of G2-like genes in response to abiotic stress (cold, drought and salt) based on RNA-seq data was also examined. Further studies exhibited that silencing SIG/k16 would reduce the drought stress resistance of tomato plants. We aimed to identify genes that are important to stress resistance and thus lay a theoretical foundation for resistance improvement in tomato by providing genetic resources to accelerate the process of tomato breeding.

\section{Results}

\section{Identification and Physical Property Analysis of Putative G2- Like Proteins in Tomato}

A total of 66 G2-like proteins (designated SIGIk1 to S/GIk66) were identified using the bioinformatics approach. Their basic physical information is presented in Table S2. The predicted molecular weights ranged from 20316.24 Da (SIGIk60) to 77633.43 Da (SIG/k63). Consistent with the molecular weights, SIGIk60 (174 aa) had the shortest sequence and SIGIk63 (708 aa) had the longest. The isoelectric point 
varied from 4.83 (S/G/k34) to 10.07 (S/G/k46), and the predicted aliphatic indices varied from 55.85 (SIGIk21) to 90.43 (SIGIk58). The instability indices varied from 33.02 (SIG/k8) to 63.18 (SIG/k61), and the instability indices of $S / G / k 8 / 9 / 22 / 30 / 43 / 62$ were lower than 40 , indicating that these proteins were relatively stable. The hydrophobicity indices ranged from -1.003 (SIG/k28) to -0.29 (SIG/k14).

\section{Sequence Alignment And Phylogenetic Analysis}

The phylogenetic relationships of the G2-like proteins of tomato were classified into five groups according to their groupings with maize $\mathrm{G} 2$-like proteins and motif analysis of tomato G2-like proteins (Text S1 and Text S2). Groups I to V contained 12, 6, 4, 18 and 26 G2-like proteins, respectively (Figure $1 \mathrm{~A}$ ). Multiple sequence alignments (Figure 1B) revealed that the genes were conserved across at least two regions of the HLH structure of the Myb-like domain: the first helix contained the initial sequence PELHRR (except in SIGIk46), and the second helix contained NI/VASHLQ. In addition, most SIG/ks in group $\nabla$ had a specialized conserved Myb-CC-LHEQLE domain. A separate multiple sequence analysis was also performed in each subgroup (Figure S1). In the members of groups $\otimes$ and II, the form was mostly LHR/L/H/A; PELHRR and PDLHRR were the two types of conserved domains in group $\triangle$ members; the form of PELHRR was XD/ELHD/EX in group $₫$ members; and XQ/ELHXX was the main type in group $V$ members. The VI/NASHLQ domain was more conserved than the PELHRR domain was in all the groups. Four main types were present in members of all the groups: LKSHLQ, VKSHLQ, IKSHLQ and VASHLQ. On the basis of the results of the analysis of each group, we summarized the types of G2-like conserved domains (Table S3).

\section{Motif Analysis and Gene Structure of Tomato G2-like Genes}

Fifteen putative motifs whose lengths varied from 13 to 50 amino acids were identified via the MEME website (Table S4) as representing the structure of all G2-like proteins. The results of the motif analysis are shown in Fig. 2B. Each protein sequence includes a different number of motifs (1 7), and each motif is present only once, with the exceptions of motif $13 /$ motif 2 and motif 6 , which were present twice in S/GIk6 and S/GIk8, respectively. Most G2-like proteins (83.33\%) contained motif 1 and motif 2, which corresponded to the myb SHAQKYF domain based on Pfam database and Conserved Domain Database (CDD). We also found that the PLN03162 superfamily domain also contained motif 1 and motif 2. SIGIk62 and SIGIk46, which corresponded to the myb SHAQKYF domain and PLN03162 superfamily domain, contained only motif 2 and motif 11 , respectively. The same motif compositions correspond to different domains, or different motif compositions correspond to the same domain, suggesting functional diversity. Details of the CDD results are showed in Table S5. The G2-like proteins clustered in the same group of the phylogenetic tree contained a similar motif composition besides $S / G / k 46 / 62$, showing that they were highly conserved. For example, motif 15 was present only in group $\otimes$ members, which had very similar sequences. $S / G / k 6 / 7 / 8 / 9$, which were formed from tandem duplications, also presented similar motif compositions. The function of the majority of these motifs needs to be further studied. 
Studying the introns and exons of tomato, which were determined by the alignment of G2-like genes, would give more insight into the evolution of the G2-like family members in tomato. Intron and exon predictions are shown in Fig. 2C, and the sequences of the G2-like genes are shown in Text S1. The number of exons varied from 1 to 11 . More than half of the G2-like genes $(62,93.94 \%)$ had four or more exons, and only 4 genes $(6.06 \%)$ had three or fewer exons. The conserved regions of all of the tandemly duplicated genes and segmentally duplicated genes presented similar exon distributions. Overall, the phylogenetic analysis results, motif composition and similar gene structure of the G2-like members in the same group provided reliable results for group classification.

\section{Chromosomal Location and Gene Duplication Events of G2-like Genes in Tomato}

S/GIks were unevenly distributed across the 12 tomato chromosomes, and the locations of most $S / G / k s$ were on the proximal or distal ends of the tomato chromosomes. The number of $S / G / k s$ per chromosome ranged from 2 to 9 (Chr10 had 9 genes; Chr03 only had 2 genes). As shown in Fig. 3, Chr01, which is the longest chromosome, contained only 3 genes. There was no significant positive correlation between chromosome length and gene number.

Genome duplication events, which are usually divided into three types (tandem duplications, segmental duplications and transposition events), occurred during plant evolution [33-35]. Tandem duplications are defined as chromosomal regions that are less than $200 \mathrm{~kb}$ in length and contain two or more genes [36]. There were 4 genes (S/G/k6/7/8/9) located on Chr02, which resulted from tandem duplications, that formed 1 tandem duplication event region. Using BLASTP and MCScanX, we also found 9 segmental duplications (18 G2-like genes in total) events (Figure 3 and Table S6). Taken together, these results showed that some $S / G / k s$ may have arisen via gene duplication.

\section{Analysis Of Cres}

To further determine the potential function of G2-like genes in response to abiotic stress, the CREs within the sequences $2 \mathrm{~kb}$ upstream from the translation start site of the G2-like genes were searched within the PlantCare database. Analysis of the promoters of $S / G / k s$ in tomato revealed that all family members contained light-responsive elements and two core elements-the CAAT box and TATA box. Detailed elements are displayed in Table S7 and Fig. 4. There are also significant differences in the number of CREs among the promoters of the different members of the G2-like gene family. As shown in Fig. 4, the promoters of SIGlk18 contained the most kinds of CREs (13), while SIGIk34 contained only three kinds of CREs. Only 20 SIGlks did not have any abiotic stress response elements, while the other SIGlks contained at least one abiotic stress element, which indicated that the expression of more than half of the G2-like genes was related to abiotic stress. In addition, we found that $52 \mathrm{~S} / G / k s(78.79 \%)$ had two or more hormone induction elements and that $S / G / k 47$ contained all five hormone induction elements, such as ABA-, IAA-, GA-, JA- and SA-induction elements. Regardless, analysis of the CREs showed that the number and distribution of CREs and that CREs in the same subgroup were not similar, which indicated that each 
S/Glks are regulated by different combinations of TFs and that the expression of S/G/ks could be induced in response to different hormones and abiotic stresses.

\section{G2-like Gene Expression Patterns in Response to Different Abiotic Treatments}

To explore the G2-like genes that respond to three different abiotic stresses, we downloaded RNA-seq data from the NCBI database; data for S/GIk4/9/43/57/58 were not found in the RNA-seq database. Nonetheless, the information was presented in the form of a heatmap (Table S8 and Fig. 6). As shown in Fig. 5, there were 40 genes expressed in response to cold stress, 42 genes expressed in response to drought stress and 41 genes expressed in response to salt stress. We found that the expression of S/Glk22/44/25/59/29/56/24 was high in response to the three different stress treatments. The expression of SIGlk56 increased but then decreased after cold and drought stress treatment. Moreover, the expression pattern of $S / G / k 36$ decreased but then increased after drought and salt stress treatment, while the opposite occurred after cold stress treatment. These results indicated that some G2-like genes are involved in abiotic stress responses. We defined G2-like genes DEGs whose expression levels changed more than or equal to twofold than $0 \mathrm{~h}$ (and when $\mathrm{P}<0.05$ ). A total of $53.03 \%$ of $G 2$-like genes, including 5 cold stress-related genes, 22 drought stress-related genes and 27 salt stress-related genes, were differently expressed. As shown in Fig. 6A, more than half of the DEGs (21 genes) responded to one stress treatment, and only 11 genes responded to two stresses. Four genes, S/Glklk11/20/26/62, were expressed in response to all the stresses. The detailed gene list is shown in Fig. 6B. The DEGs that responded to only one abiotic stress or that differed from the other two genes, are shown in Fig. 6C. The results showed that the expression of half of the drought specific-related genes was upregulated. In contrast, the expression of most salt specific-related genes was downregulated. Interestingly, we also found that the expression of $S / G / k 44 / 21 / 1$ and $S / G / k 46 / 22 / 65 / 36$ was up-/down-regulated under both drought and salt stresses respectively.

\section{Expression of Tomato G2-like Genes in Response to Abiotic Stress and Hormone Treatments}

To verified the expression data from RNA-seq and explore whether the expression of $S / G / k s$ is affected by hormone treatments, we randomly selected 11 tomato G2-like genes from among the 36 DEGs to investigate through qRT-PCR the transcript levels of these 11 genes in response to different treatments. Detailed expression patterns of these G2-like genes are shown in Fig. 7. The expression of these 11 genes generally corresponded to RNA-seq results, indicating the RNA-seq results was reliable. We also found that the expression of these $S / G / k s$ could be induced by at least 4 different abiotic stress and hormones treatments after $24 \mathrm{~h}$ and $12 \mathrm{~h}$. Interestingly, we also found that the expression of some S/G/ks was induced/repressed by one treatment. For instance, the expression levels of all SIGlks except SIG/k36 were upregulated under JA and IAA treatment, while the expression level of most $S / G / k s$ were downregulated under drought and ABA treatment. The expression levels of seven SIGIks (S/Glk61/16/38/55/53/54/64) were first upregulated but then were downregulated under GA treatment, and the expression of S/G/k16 exhibited the same dynamic pattern under abiotic stresses. Several genes, such as $S / G / k 38 / 55$, exhibited 
opposite expression patterns under different treatments. Taken together, these results showed that $S / G / k s$ were influenced by most of the applied abiotic stress and hormone treatments.

\section{GO Enrichment and KEGG Enrichment Analysis of G2-like DEGs}

To further determine the function of the $36 \mathrm{DEGs}$, GO enrichment and KEGG enrichment analysis was performed. The results of GO analysis can be generally divided into three categories: BP, CC and MF. We found that $87 \mathrm{GO}$ terms were enriched for $31 \mathrm{DEGs}$, excluding S/GIk22/23/38/14/34 and the detailed information was displayed in Figure S2. Biological regulation and cellular process were dominant in the BP category. CC clusters contained two subcategories, cell and organelle, with 31 DEGs, and binding was the only subcategory among the MF category. The top 20 terms for enrichment analysis with the whole genome as the background according to $P$ value are shown in Fig. 8A. With the exception of DNA binding, the remaining of 19 terms belonged to BP groups. Thirty-five DEGs (SIG/k62 was excluded) were mapped to $3 \mathrm{KEGG}$ pathways (P value<0.05): plant hormone signal transduction; arginine biosynthesis; and alanine, aspartate and glutamate metabolism (Figure 8B and Table S9). Therefore, we divided the annotated DEGs into two groups: $31 \mathrm{DEGs}$ in the plant hormone signal transduction pathway were assigned to group 1 while 4 DEGs ( $S / G / k 25 / 44 / 45 / 50)$ related to amino acid metabolism were assigned to group 2. We further statistically analyzed the expression patterns of these genes under each abiotic stress in the two groups. We found that the expression patterns of DEGs in the same group were different. In group 1, the proportion of genes whose expression was downregulated was higher than the genes whose expression upregulated under drought and salt stress treatment, while the number of genes whose expression was upregulated was far greater than the number of genes whose expression was downregulated under cold stress treatment. In group 2, the proportions of genes whose expression was downregulated and the genes whose expression was upregulated were equal, and the expression of only one gene was upregulated. However, the expression of none of them changed under cold stress.

\section{Silencing SIGIk16 reduced drought tolerance in tomato plants}

Based on the previous study (the S/Glks expression under different treatments and RNA-seq databases under abiotic treatments), SIGIk16 which is defined as drought-specific related DEG was selected for further analysis under drought stress. Figure S3 showed the expression level of S/G/k16 in the infected tomato seedlings and we chose the plants (4 seedlings) whose SIGlk16 expression level was less $50 \%$ than CK for further test. As shown in Fig. 9A, the growth status of all plants was in similar before abiotic treatment, and the wilting of all plants was increased with increasing drought treatment time. Noteworthy, the leaves of $S / G / k 16$-silenced plants began to wilt and the stem began to curl after $3 \mathrm{~h}$ drought treatment, while other plants only exhibited a slight curled stem. After $12 \mathrm{~h}$ later, the leave of S/Glk16-silenced plants showed wilting and drying more severe than the other two groups, although the above phenomena increased in severity in all the plants. The activity of SOD, POD and the content of MDA and Pro were measured for all plants under normal and drought treatment to explain the decreased drought tolerance in SIGIk16-silenced plants. After drought treatment, the activity of SOD and POD and the content of MDA increased in all plants. Under drought treatment, the activities of SOD and POD in S/GIk16-silenced plants 
were lower than that in the other two groups (Figure 9B, C). However, the MDA contents in SIGIk16silenced plants were higher than others after drought treatment (Figure 9D). Interestingly, we also found that the Pro contents in detached leaves of S/GIk16-silenced plants were decreased after drought treatment and lower than CK and CK-TRV2.

\section{Discussion}

In this study, 66 nonredundant G2-like genes, which were designated SIGIk1 to SIGIk66 on the basis of their chromosomal location, were identified and divided into five groups (I to $\mathrm{V}$ ) according to their grouping with the sequences of those of Arabidopsis and maize. A previous study revealed that there are 59 and 45 Glk proteins in maize and sorghum, respectively, and the maize Glk proteins could be divided into seven groups [27]. We first obtained seven subgroups by phylogenetic analysis with maize Glk proteins, and then three subgroups whose members had a similar motif distribution were merged into one subgroup. Thus, the tomato SIGlk proteins were ultimately divided into five subgroups. The SIGlks belonging to the same subgroups showed strong resemblance in terms of their structure and number of exons and introns, and they were adjacent in the phylogenetic tree. These consistent results suggested that the $S / G / k s$ groupings were relatively reliable.

We aligned all 66 G2-like proteins via Jalview. In Figure 1B shows that the SIGlks were conserved to a certain extent. We then aligned the 66 sequences according to their different groups. With respect to the PELHRR conserved domain, we observed that $L$ and $H$ were highly conserved in all the groups, and the PELHRR domain was present in various types. In addition, SHLQ was very conserved in the VI/NASHLQ domain, existing as only 8 variants, as shown in Table S3. These results suggested that the PELHRR domain may play a role in functional differentiation. The PELHRR and VI/NASHLQ domains were derived on the basis of an analysis of maize G2-like (ZmGlk) proteins, and we observed that these two conserved domains in SIGlks were similar to those in ZmGlks, but they also differed in their own composition (Liu et al. 2016). Therefore, we deduced that even if the proteins from different species represented the same type of TF, their structure probably varied. The members of groups $\nabla$ and $\nabla$ varied more, and hence, those members may be involved in more biological functions than those of the other groups.

According to the chromosomal location map, S/Glks are widely and unevenly distributed across all 12 chromosomes of tomato. Gene duplication is a significant means for gene family expansion during the evolution of plant genomes. Among the $S / G / k s, 12$ duplication events have occurred: 9 segmental duplications and 3 tandem duplications (Figure 3). Segmental duplications were obviously the main way in which the SIGlkgene family expanded in tomato. Many studies have shown that segmental duplication is more common than tandem duplication [27,37-39], so the former may play an important role in the long-term evolutionary process. Some researchers believe that segmental duplication occurs regularly in slowly evolving gene families [27]. Taken together, these results indicated that tomato G2-like genes have probably been relatively conserved throughout the evolutionary process. 
Many adverse conditions such as low temperature, salinity and drought, act as limiting factors in the process of plant growth and development. Many studies have also indicated that hormones are necessary for plant responses to biotic and abiotic stresses [40-43]. Hence, we analyzed the expression of 11 select tomato G2-like genes under three abiotic stresses and five hormone treatments. Previous studies have shown that orthologous genes in different species have conserved ancestral gene functions, but paralogous genes have distinct functions due to gene duplication [44, 45]. Interestingly, in a previous study, we found that the expression of $S / G / k 38$ and its ortholog in maize, ZmGIk11, exhibited opposite patterns in response to cold and drought stresses [27], suggesting that $S / G / k s$ may have lost or gained new functions during evolution. In the present study, the expression of SIGIk45 was induced under IAA and JA treatment and repressed under cold, salt, drought, ABA and GA treatment. However, the expression profiles of $S / G / k 65$ differed from those of $S / G / k 45$, although both segmentally duplicated genes (Figure 7). The expression of most the selected genes was in response to IAA and JA. Studies have also shown that AtGlk1 is related to $\mathrm{SA}$ and $\mathrm{JA}$ signaling in disease defense, including that against Hyaloperonospora arabidopsis ( $\mathrm{Hpa}$ ) Noco2 and Botrytis cinerea [46-51]. In addition, OsGlk1 may participate in resistance to pathogen invasion [48], and AtGlks may be useful in tolerance to Cucumber mosaic virus [24]. Therefore, we conclude that $S / G / k s$ may play important roles in disease resistance. Further study of these genes might allow us to obtain more stress-resistant and disease-resistant tomato varieties.

It has been reported that TFs can integrate multiple CREs to regulate gene expression [52]. In the present study, CRE analysis showed that the promoter region of SIGlks have many abiotic- and hormone-related elements, such as MBS, ABRE, GARE-motif and LTR. Interestingly, we found that the expression of S/GIks is not strongly correlated with CREs. For example, we found that $S / G / k 3 / 64 / 65$ contain IAA-esponsive elements, so we surmised that the expression of $S / G / k 3 / 64 / 65$ could be induced by IAA treatment, which is consistent with our qPCR findings. Conversely, the promoters of $S / G / k 16 / 38 / 45 / 61 / 53$, which contain no IAA-responsive elements, could also be induced by IAA treatment (Figures 4 and 7). Thus, we speculated that CREs with unknown functions are related to IAA. We also found that different numbers of the same CRE would affect the expression level of $S / G / k s$. For example, the expression level of $S / G / k 53$ was more significantly induced than that of $S / G / k 16$ was under GA treatment; the promoter region of SIGIk53 contained three GA-responsive elements, whereas that of SIGIk16 contained only one (Fig. 4 and 7).

On the basis of RNA-seq data, the number of DEGs and specific responsive of S/Glks was not vastly different under drought and salt stresses, while the fewest number of genes were differentially expressed in response to cold stress. These results indicated that G2-like genes play an important role in drought and salt responses. Thirty-five DEGs were enriched in K004075 which is related to zeatin biosynthesis. Zeatin is a plant growth regulator, which can promote cell growths, prevent chlorophyll and protein degradation, maintain cell vitality and delay plant senescence. Thus, we suspected that G2-like genes could regulate the content of zeatin in plants, so that the tomato plants could adapt to the abiotic stresses. 
In the present study, we found that silencing S/GIk16 in tomato exhibited reduced drought stress tolerance by earlier wilting and variation of corresponding physiological indexes under drought stress, which indicated that $S / G / k 16$ is a positive regulator in drought stress. It is known that the activities of SOD and POD play an important role to protect cell membranes from ROS attack [53]. The contents of MDA and Pro could reflect the damage degree of cell and help plants adapt osmotic stress [54, 55]. In our study, we found that the activities of SOD and POD and Pro contents in S/GIk16-silenced plants were lower than the other two groups under drought stress while the SIGIk16-silenced plants had higher MDA contents, which suggesting that silencing SIGlk16 would reduce the stability of cell membrane and the activity of peroxidases and finally reduced their drought tolerance.

\section{Conclusions}

In this study, 66 G2-like genes in the tomato genome were characterized and classified into five groups with distinct structures and motif compositions. These genes were unevenly distributed across the 12 tomato chromosomes, and 21 S/Glks were considered duplicate genes. qRT-PCR and RNA-seq data showed that most G2-like genes could respond to different abiotic stress and hormone treatments, and KEGG analysis revealed that G2-like proteins may be related to zeatin biosynthesis. Finally, S/G/k16 was defined as a positive regulator under drought stress. Taken together, these results provide comprehensive information to further study the function of G2-like gene family members in response to abiotic and biotic stresses.

\section{Methods}

\section{Identification of G2-like Genes and Construction of Phylogenetic Trees}

The tomato sequence file SL3.0 was downloaded from the Solanaceae Genomics Network (SGN) (http://solgenomics.net).Two Arabidopsis G2-like protein sequences (AT2G20570.2 and AT5G44190.1) from The Arabidopsis Information Resource (TAIR 9) (https://www.arabidopsis.org/) were used as query sequences to identify G2-like sequences within tomato protein databases by BLASTP (E-value: 1e-5). To further test the obtained tomato sequences, we compared them with G2-like gene family classification criteria, in which the members of G2-like TFs should include G2-like domains and exclude Response reg domain according to the Plant Transcription Factor Database (PIn TFDB 3.0) (http://plntfdb.bio.unipotsdam.de/v3.0/), via the HMMER program (http://www.ebi.ac.uk/Tools/hmmer/). We then used SMART (http://smart.embl-heidelberg.de/) to further verify the identities of all the proteins. Finally, the physical parameters of all the confirmed amino acid sequences were estimated using the online software ExPASy (http://web.expasy.org/protparam/). Protein and nucleic acid sequences of maize were acquired from a previous publication [27].

Multiple protein sequence alignments of tomato and maize were executed using ClustalX [56]. Afterward, an unrooted phylogenetic tree was constructed with MEGA 7.0 software [57] via the maximum likelihood method with the Jones-Taylor-Thornton (JTT) model and 1000 bootstrap replicates. G2-like proteins were 
classified according to the topology and bootstrap values of the phylogenetic tree. The G2-like protein sequences of tomato and maize are listed in Texts S1 and S2.

\section{Gene Structure Analysis, Motif Analysis and Cis-regulatory Element (CREs) Analysis, Chromosomal Location and Gene Duplication Events of G2-like Genes in Tomato}

Gene structure analysis included the prediction of introns and exons, which were analyzed via SGN (http://solgenomics.net). The conserved motifs of the tomato G2-like proteins were evaluated via online MEME software (http://meme-suite.org/). The number of motifs found in MEME was limited to 15, the positions of the conserved domains were predicted using Jalview software, the exon-intron structures were displayed by TBtools [58]. The CREs were selected from the $2000 \mathrm{bp}$ upstream sequences of the start codon of all SIGIks via the PlantCARE database (http://bioinformatics.psb.ugent.be/webtools/plantcare/html/) [59].

Chromosomal position information was obtained from the SGN (http://solgenomics.net), and all SIGIks were mapped onto the tomato chromosomes by Circos [60]. The Multiple Collinearity Scan Toolkit (MCScanX), with the default parameters, was used to determine the gene duplication landscape [61]. A syntonic map was subsequently displayed by TBtools [58].

\section{RNA-seq Analysis and Gene Expression Heatmap of G2-like Genes}

The expression patterns of G2-like genes in response to cold, drought and salt stresses were revealed by analyzing the RNA-seq data. The original RNA-seq data were obtained from the NCBI database, of which the accession numbers with respect to the response to cold, drought and salt stresses were GSE148887, GSE148530 and GSE148353 respectively, and the RNA-seq data of Micro-Tom (CK) was only used. The fragments per kilobase per Million (FPKM)-normalized fragment data of significantly expressed genes $(F P K M>1)$ was $\log _{2}$ transformed, and the expression patterns of $\mathrm{G} 2$-like genes were visualized by a heatmap via Multiple Experiment Viewer 4.0.

\section{Plant Material And Treatments}

The tomato (Solanum lycopersicum) cultivar Micro-Tom and Money Maker (MM), which was provided by the Tomato Research Institute of Northeast Agricultural University, was used in this study. Seedlings were grown under a day: night temperature of $20 \sim 25^{\circ} \mathrm{C}: 13 \sim 15^{\circ} \mathrm{C}$ at $45 \%$ relative humidity under a $13: 11 \mathrm{~h}$ light: dark photoperiod. Five-week-old Micro Tom plants were irrigated with $15 \%$ polyethylene glycol (PEG) 6000 and $200 \mathrm{mM} \mathrm{NaCl}$ for drought and salt treatments, respectively; for cold treatment, tomato plants were placed in a $4^{\circ} \mathrm{C}$ growth chamber. Leaf samples from plants subjected to abiotic stress treatments were collected after $0,3,6,12$ and $24 \mathrm{~h}$ of stress imposition. For phytohormone treatments, solution of $100 \mu \mathrm{M}$ abscisic acid (ABA) and jasmonic acid (JA), $400 \mu \mathrm{M}$ salicylic acid (SA), $450 \mathrm{mg} / \mathrm{L}$ indoleacetic acid (IAA) and $300 \mathrm{mg} / \mathrm{L}$ gibberellic acid (GA) were sprayed onto tomato plants in accordance which complied with the requirements. Leaf samples were collected at $0,3,6,12 \mathrm{~h}$ after the phytohormone 

isolation.

\section{RNA Extraction, cDNA Synthesis and Quantitative Real Time PCR (qRT-PCR) Analysis}

Total RNA was extracted from the samples using TRIzol. First-strand cDNA was synthesized using $1 \mu$ of total RNA via a transcript kit purchased from Beijing TransGen Biotech. RT-qPCR was carried out using AceQ qPCR (SYBR Green Master Mix) in conjunction IQ5. The Actin gene (Solyc11g005330.1.1) was used as an internal control. For each qRT-PCR, $1 \mu \mathrm{l}$ of diluted CDNA, $0.5 \mu$ l each of forward primer and reverse primer, $10 \mu \mathrm{l}$ of SYBR and $8 \mu \mathrm{l}$ of $\mathrm{dd}_{2} \mathrm{O}$ were used in a $20 \mu \mathrm{l}$ reaction. The reaction was carried out as follows: $95^{\circ} \mathrm{C}$ for $5 \mathrm{~min}$, followed by 40 cycles of $94^{\circ} \mathrm{C}$ for $5 \mathrm{~s}, 60^{\circ} \mathrm{C}$ for $15 \mathrm{~s}$ and $72^{\circ} \mathrm{C}$ for $10 \mathrm{~s}$; three biological replicates were included. The $2^{-\Delta \Delta C t}$ method was used for quantification and Actin as a reference gene. Information about the primers is shown in Table S1. Three replicates were necessary for each time point.

Gene Ontology (GO) and Kyoto Encyclopedia of Genes and Genomes (KEGG) Enrichment Analysis of Differentially Expressed Genes (DEGs) of G2-like TFs

GO enrichment analysis of DEGs was performed using the GOseq R package. The GO analysis involved three categories: molecular function (MF), biology progress (BP) and cellular component (CC). We inferred the behavior of cells or organisms on the basis of the genome or transcriptome of genes annotated to KEGG pathways [62], and KOBAS software was used to determine the DEGs enriched in the various KEGG pathways [63]. The results of the GO and KEGG analyses are shown via bubble chart.

\section{Generation of SIGIk16-silenced plants and phenotypic observation under drought stress}

The SIGIk16 fragment was amplified from cDNA made from MM RNA (leave samples) via specific primers (Table S1) designed in accordance with N Fernandez-Pozo, HG Rosli, GB Martin and LA Mueller [64]. The target sequence of SIGlk16 was then cloned into tobacco rattle virus RNA2 (TRV2) via ClonExpress II One Step Cloning Kit (Vazyme, China). To obtained S/G/k16-silenced plants, the fusion plasmid TRV2-SIGIk16 was introduced into Agrobacterium tumefaciens GV3101 which was then infiltrated into leaves of five fully expanded leaves of MM seedlings according to the method [65]. The expression levels of $S / G / k 16$ in infiltrated seedlings were determined by qRT-PCR, and the lines with the expression levels less than $50 \%$ than CK were used for further drought stress treatment.

For drought-resistance test, SIGIk16-silenced seedlings, CK and CK-TRV2 grown in Hoagland's solution for $24 \mathrm{~h}$ and then transferred into $15 \%$ PEG6000 solution for $12 \mathrm{~h}$. We observed the phenotype of the plants at $0,3,12 \mathrm{~h}$ after drought stress treatment.

\section{Physiological Measurements}


Leaf samples were collected from CK, CK-TRV2 and SIGIk16-silenced seedlings before and after drought stress treatment. We used the corresponding kits (Keming, China) to measure the contents of proline (Pro), malondialdehyde (MDA) and determine the activities of superoxide dismutase (SOD) and peroxidase (POD) according to the manufacturer's protocol. Three independent biological replicates were used for all measurements.

\section{Statistical analysis}

Statistically significant differences between CK, CK-TRV2 and S/Glk16-silenced plants in terms of the measured parameters were tested by Ducan's multiple range tests. Different lowercase letters indicate significant differences $(P<0.05)$. Bars are SE $(n=3)$.

\section{Abbreviations}

Golden 2-Like: G2-like; Transcription factors: TFs; Conserved Domain Database: CDD; CREs: cis-regulatory elements; SGN: Solanaceae Genomics Network; TAIR: The Arabidopsis Information Resource; PIn TFDB 3.0: Plant Transcription Factor Database; JTT model: Jones-Taylor-Thornton model; MCScanX: Multiple Collinearity Scan Toolkit; FPKM: Fragments per kilobase per Million; Money Maker: MM; PEG:

Polyethylene glycol; ABA: Abscisic acid; JA: Jasmonic acid; SA: Salicylic acid; IAA: Indoleacetic acid; GA: Gibberellic acid; qRT-PCR: Quantitative real; GO: Gene Ontology; KEGG: Kyoto Encyclopedia of Genes and Genomes; DEGs: Differentially Expressed Genes; MF: Molecular function; BP: Biology progress; CC: Cellular component; TRV2: Tobacco rattle virus RNA2; Pro: Proline; MDA: Malondialdehyde; SOD: Superoxide dismutase; POD: Peroxidase

\section{Declarations}

\section{Ethics approval and consent to participate}

Not Applicable.

\section{Consent for publication}

Not applicable.

\section{Availability of data and material}

The web link to the RNA-seq database are as follows:

Comparative transcriptome analysis between two tomato materials under cold stress

https://www.ncbi.nlm.nih.gov/geo/query/acc.cgi?acc=GSE148887

Comparative transcriptome analysis between two tomato materials under drought stress 
https://www.ncbi.nlm.nih.gov/geo/query/acc.cgi?acc=GSE148530

Comparative transcriptome analysis between two tomato materials under salt stress

https://www.ncbi.nlm.nih.gov/geo/query/acc.cgi?acc=GSE148353

We declare that the dataset(s) required to reproduce the results of this article are included in the article and additional file(s) available in the journal webpage.

\section{Competing interest}

Not applicable.

\section{Funding}

This work was supported by the National Natural Science Foundation of China (Grant No. 32072589); "Hundred and ten million" Project Science and Technology Major Special Subject of Heilongjiang Province (Grant No. 2019ZX16B02); Postdoctoral science foundation of Heilongjiang province (Grant No. LBHZ19046); Natural Science Funds for Distinguished Young Scholar of Heilongjiang Province (Grant No. YQ2021C013); Program for Backbone of Academic Research of Scholar Project of Northeast Agricultural University (Grant No. 20XG28).

\section{Authors' contributions}

Conceptualization, T.Z. and X.X.; Data curation, Z.W.; Formal analysis, Z.W.; Funding acquisition, T.Z. and X.X.; Investigation, Z.W.; Methodology, Z.W., H.Y., H.Z., J.J., T.Z. and X.X.; Project administration, T.Z. and X.X.; Resources, H.Z., Y.W. and Q.L.; Supervision, JFu.L.; Validation, JFang.L., X.S., T.W. and T.P.; Writing original draft, Z.W.; Writing - review \& editing, T.Z. and X.X.. All authors have read and approved the final manuscript, and ensure that this is the case.

\section{Acknowledgements}

Not applicable.

\section{References}

1. Gao J, Peng H, He X, Luo M, Zhang Z: Molecular phylogenetic characterization and analysis of the WRKY transcription factor family responsive to Rhizoctonia solani in maize. Maydica 2014, 59(1):3241.

2. Ling J, Jiang W, Zhang Y, Yu H, Mao Z, Gu X, Huang S, Xie B: Genome-wide analysis of WRKY gene family in Cucumis sativus. BMC Genomics 2011, 12(1):1-20.

3. Song A, Gao T, Wu D, Xin J, Chen S, Guan Z, Wang H, Jin L, Chen F: Transcriptome-wide identification and expression analysis of chrysanthemum SBP-like transcription factors. Plant Physiology and 
Biochemistry 2016, 102:10-16.

4. Kou X, Wang S, Wu M, Guo R, Xue Z, Meng N, Tao X, Chen M, Zhang Y: Molecular Characterization and Expression Analysis of NAC Family Transcription Factors in Tomato. Plant Molecular Biology Reporter 2014, 32(2):501-516.

5. Junya M, Kazuo S, Kazuko Y-S: AP2/ERF family transcription factors in plant abiotic stress responses. Biochimica et Biophysica Acta (BBA) - Gene Regulatory Mechanisms 2012, 1819(2):8696.

6. Riechmann JL, Heard J, Martin G, Reuber L, Jiang C-Z, Keddie J, Adam L, Pineda O, Ratcliffe OJ, Samaha RR et al: Arabidopsis Transcription Factors: Genome-Wide Comparative Analysis Among Eukaryotes. Science 2000, 290(5499):2105-2110.

7. Wang J, Zheng C, Shao X, Hu Z, Li J, Wang P, Wang A, Yu J, Shi K: Transcriptomic and genetic approaches reveal an essential role of the NAC transcription factor SINAP1 in the growth and defense response of tomato. Horticulture Research 2020, 7(1).

8. Kaplan-Levy RN, Brewer PB, Quon T, Smyth DR: The trihelix family of transcription factors - light, stress and development. Trends in Plant ence 2012, 17(3):163-171.

9. Jenkins MT: A Second Gene Producing Golden Plant Color in Maize. The American Naturalist 1926, 60(670):484-488.

10. Hall LN, Rossini L, Langdale CJA: GOLDEN 2: A Novel Transcriptional Regulator of Cellular Differentiation in the Maize Leaf. The Plant Cell 1998, 10(6):925-936.

11. Imamura A, Hanaki N, Nakamura A, Suzuki T, Taniguchi M, Kiba T, Ueguchi C, Sugiyama T, Mizuno T: Compilation and Characterization of Arabiopsis thaliana Response Regulators Implicated in His-Asp Phosphorelay Signal Transduction. Plant Cell Physiology 1999, 40(7):733-742.

12. Wykoff DD, Grossman AR, Weeks DP, Usuda H, Shimogawara K: Psr1, a nuclear localized protein that regulates phosphorus metabolism in Chlamydomonas. Proceedings of the National Academy of Sciences 1999, 96(26):15336-15341.

13. Rossini L, Cribb L, Martin DJ, Langdale JA: The Maize Golden2 Gene Defines a Novel Class of Transcriptional Regulators in Plants. The Plant Cell 2001, 13(5):1231-1244.

14. Hosoda K, Imamura A, Katoh E, Hatta T, Tachiki M, Yamada H, Mizuno T, Yamazaki T: Molecular Structure of the GARP Family of Plant Myb-Related DNA Binding Motifs of the Arabidopsis Response Regulators. The Plant Cell 2002, 14(9):2015-2029.

15. Bravo-Garcia A, Yasumura Y, Langdale JA: Specialization of the Golden2-like regulatory pathway during land plant evolution. New Phytologist 2009, 183(1):133-141.

16. Waters MT, Moylan EC, Langdale JA: GLK transcription factors regulate chloroplast development in a cell-autonomous manner. The Plant Journal 2008, 56(3):432-444.

17. Brand A, Borovsky Y, Hill T, Rahman KAA, Bellalou A, Van Deynze A, Paran I: CaGLK2 regulates natural variation of chlorophyll content and fruit color in pepper fruit. Theoretical and Applied Genetics 2014, 127( 10):2139-2148. 
18. Fitter DW, Martin DJ, Copley MJ, Scotland RW, Langdale JA: GLK gene pairs regulate chloroplast development in diverse plant species. Plant Journal 2002, 31(6):713-727.

19. Powell ALT, Nguyen CV, Hill T, Cheng KL, Figueroa-Balderas R, Aktas H, Ashrafi H, Pons C, FernándezMuñoz R, Vicente A et al: Uniform ripening Encodes a Golden 2-like Transcription Factor Regulating Tomato Fruit Chloroplast Development. Science 2012, 336(6089):1711-1715.

20. Langdale JA, Kidner CA: Bundle sheath defective, a mutation that disrupts cellular differentiation in maize leaves. Development1994, 120(3):673-681.

21. Langdale JA, Nelson T: Spatial regulation of photosynthetic development in C4 plants. Trends in Genetics 1991, 7(6):191.

22. Nguyen CV, Vrebalov JT, Gapper NE, Zheng Y, Zhong S, Fei Z, Giovannoni JJ: Tomato GOLDEN2-LIKE Transcription Factors Reveal Molecular Gradients That Function during Fruit Development and Ripening. The Plant Cell 2014, 26(2):585-601.

23. Kobayashi K, Sasaki D, Noguchi K, Fujinuma D, Komatsu H, Kobayashi M, Sato M, Toyooka K, Sugimoto K, Niyogi KK et al: Photosynthesis of Root Chloroplasts Developed in Arabidopsis Lines Overexpressing GOLDEN2-LIKETranscription Factors. Plant and Cell Physiology 2013, 54(8):13651377.

24. Han XY, Li PX, Zou LJ, Tan WR, Zheng T, Zhang DW, Lin HH: GOLDEN2-LIKEtranscription factors coordinate the tolerance to Cucumbermosaic virus in Arabidopsis. Biochemical and Biophysical Research Communications 2016, 477(4):626-632.

25. Savitch LV, Subramaniam R, Allard GC, Singh J: The GLK1 'regulon' encodes disease defense related proteins and confers resistance to Fusarium graminearum in Arabidopsis. Biochemical and Biophysical Research Communications 2007, 359(2):234-238.

26. Schreiber KJ, Nasmith CG, Allard G, Singh J, Subramaniam R, Desveaux D: Found in Translation: High-Throughput Chemical Screening in Arabidopsis thaliana Identifies Small Molecules That Reduce Fusarium Head Blight Disease in Wheat. Molecular plant-microbe interactions 2011, 24(6):640-648.

27. Liu F, Xu Y, Han G, Zhou L, Ali A, Zhu S, Li X: Molecular Evolution and Genetic Variation of G2-Like Transcription Factor Genes in Maize. PLOS ONE 2016, 11(8):e0161763.

28. Nagatoshi Y, Mitsuda N, Hayashi M, Inoue S-i, Okuma E, Kubo A, Murata Y, Seo M, Saji H, Kinoshita T et al: GOLDEN 2-LIKE transcription factors for chloroplast development affect ozone tolerance through the regulation of stomatal movement. Proceedings of the National Academy of Sciences 2016, 113(15):4218-4223.

29. Gutiérrez RA, Stokes TL, Thum K, Xu X, Obertello M, Katari MS, Tanurdzic M, Dean A, Nero DC, McClung CR et al: Systems approach identifies an organic nitrogen-responsive gene network that is regulated by the master clock control gene CCA1. Proceedings of the National Academy of Sciences 2008, 105(12):4939-4944.

30. Rauf M, Arif M, Dortay H, Matallana-Ramírez LP, Waters MT, Gil Nam H, Lim P-O, Mueller-Roeber B, Balazadeh S: ORE1 balances leaf senescence against maintenance by antagonizing G2-like- 
mediated transcription. EMBO reports 2013, 14(4):382-388.

31. Yuan Y, Xu X, Gong Z, Tang Y, Wu M, Yan F, Zhang X, Zhang Q, Yang F, Hu X et al: Auxin response factor $6 \mathrm{~A}$ regulates photosynthesis, sugar accumulation, and fruit development in tomato. Horticulture Research 2019, 6(1):85.

32. Tang X, Miao M, Niu X, Zhang D, Cao X, Jin X, Zhu Y, Fan Y, Wang H, Liu Y et al: Ubiquitin-conjugated degradation of golden 2-like transcription factor is mediated by CUL4-DDB1-based E3 ligase complex in tomato. New Phytologist 2016, 209(3):1028-1039.

33. Kent WJ, Baertsch R, Hinrichs A, Miller W, Haussler D: Evolution's cauldron: Duplication, deletion, and rearrangement in the mouse and human genomes. Proceedings of the National Academy of Sciences 2003, 100(20):11484-11489.

34. Kong H, Landherr LL, Frohlich MW, Leebens-Mack J, Ma H, DePamphilis CW: Patterns of gene duplication in the plant SKP1 gene family in angiosperms: evidence for multiple mechanisms of rapid gene birth. The Plant Journal 2007, 50(5):873-885.

35. Mehan MR, Freimer NB, Ophoff RA: A genome-wide survey of segmental duplications that mediate common human genetic variation of chromosomal architecture. Human Genomics 2004, 1(5):335344.

36. Holub EB: The arms race is ancient history in Arabidopsis, the wildflower. Nature Reviews Genetics 2001, 2(7):516-527.

37. Nasim J, Malviya N, Kumar R, Yadav D: Genome-wide bioinformatics analysis of Dof transcription factor gene family of chickpea and its comparative phylogenetic assessment with Arabidopsis and rice. Plant Systematics and Evolution 2016, 302(8):1009-1026.

38. Song H, Wang P, Lin JY, Zhao C, Bi Y, Wang X: Genome-Wide Identification and Characterization of WRKY Gene Family in Peanut. Front Plant Sci 2016, 7:534.

39. Wu S, Wu M, Dong Q, Jiang H, Cai R, Xiang Y: Genome-wide identification, classification and expression analysis of the PHD-finger protein family in Populus trichocarpa. Gene 2016, 575(1):7589.

40. Ahlfors R, Lång $S$, Overmyer $K$, Jaspers $P$, Brosché $M$, Tauriainen $A$, Kollist $H$, Tuominen $H$, Belles-Boix E, Piippo $M$ et al: Arabidopsis RADICAL-INDUCED CELL DEATH1 belongs to the WWE protein-protein interaction domain protein family and modulates abscisic acid, ethylene, and methyl jasmonate responses. The Plant Cell 2004, 16(7):1925-1937.

41. Hirayama T, Shinozaki K: Research on plant abiotic stress responses in the post-genome era: past, present and future. The Plant Journal 2010, 61(6):1041-1052.

42. Lorenzo O, Solano R: Molecular players regulating the jasmonate signalling network. Current opinion in plant biology 2005, 8(5):532-540.

43. Mauch-Mani B, Mauch F: The role of abscisic acid in plant-pathogen interactions. Current opinion in plant biology 2005, 8(4):409-414.

44. Gabaldón T, Koonin EV: Functional and evolutionary implications of gene orthology. Nature Reviews Genetics 2013, 14(5):360-366. 
45. Koonin EV: Orthologs, Paralogs, and Evolutionary Genomics. Annu Rev Genet 2005, 39(1):309-338.

46. Lawton, K. A: Acquired Resistance Signal Transduction in Arabidopsis Is Ethylene Independent. Plant Cell 1994, 6(5):581-588.

47. Murmu J, Wilton M, Allard G, Pandeya R, Desveaux D, Singh J, Subramaniam R: Arabidopsis GOLDEN2-LIKE (GLK) transcription factors activate jasmonic acid (JA)-dependent disease susceptibility to the biotrophic pathogen Hyaloperonospora arabidopsidis, as well as JA-independent plant immunity against the necrotrophic pathogen Botrytis cinerea. Molecular Plant Pathology 2014, 15(2):174-184.

48. Nakamura $H$, Muramatsu $M$, Hakata $M$, Ueno O, Nagamura $Y$, Hirochika $H$, Takano $M$, Ichikawa $H$ : Ectopic Overexpression of The Transcription Factor OsGLK1 Induces Chloroplast Development in Non-Green Rice Cells. Plant and Cell Physiology 2009, 50(11):1933-1949.

49. Robert-Seilaniantz A, Grant M, Jones JDG: Hormone Crosstalk in Plant Disease and Defense: More Than Just JASMONATE-SALICYLATE Antagonism. Annual Review of Phytopathology 2011, 49(1):317-343.

50. Sanchez L, Courteaux B, Hubert J, Kauffmann S, Renault J-H, Clément C, Baillieul F, Dorey S: Rhamnolipids Elicit Defense Responses and Induce Disease Resistance against Biotrophic, Hemibiotrophic, and Necrotrophic Pathogens That Require Different Signaling Pathways in Arabidopsis and Highlight a Central Role for Salicylic Acid. Plant physiology 2012, 160(3):16301641.

51. Thaler JS, Humphrey PT, Whiteman NK: Evolution of jasmonate and salicylate signal crosstalk. Trends in Plant Science 2012, 17(5):260-270.

52. Chen WJ, Zhu T: Networks of transcription factors with roles in environmental stress response. Trends in Plant Science 2004, 9(12):591-596.

53. Shah K, Nahakpam S: Heat exposure alters the expression of SOD, POD, APX and CAT isozymes and mitigates low cadmium toxicity in seedlings of sensitive and tolerant rice cultivars. Plant Physiol Biochemistry 2012, 57:106-113.

54. Kochhar S, Kochhar VK: Expression of antioxidant enzymes and heat shock proteins in relation to combined stress of cadmium and heat in Vigna mungo seedlings. Plant Science 2005, 168(4):921929.

55. Hong Y, Zhang H, Huang L, Li D, Song F: Overexpression of a Stress-Responsive NAC Transcription Factor Gene ONAC022 Improves Drought and Salt Tolerance in Rice. Frontiers in Plant Science 2016, 7:4.

56. Thompson JD, Gibson TJ, Plewniak F, Jeanmougin F, Higgins DG: The CLUSTAL_X Windows Interface: Flexible Strategies for Multiple Sequence Alignment Aided by Quality Analysis Tools. Nucleic Acids Research 1997, 25(24):4876-4882.

57. Kumar S, Stecher G, Tamura K: MEGA7: Molecular Evolutionary Genetics Analysis Version 7.0 for Bigger Datasets. Molecular Biology and Evolution 2016, 33(7):1870-1874. 
58. Chen C, Chen H, Zhang Y, Thomas HR, Frank MH, He Y, Xia R: TBtools: An Integrative Toolkit Developed for Interactive Analyses of Big Biological Data. Molecular Plant 2020, 13(8):1194-1202.

59. Lescot M, Déhais $P$, Thijs $G$, Marchal $K$, Moreau $Y$, Van de Peer $Y$, Rouzé $P$, Rombauts S: PlantCARE, a database of plant cis-acting regulatory elements and a portal to tools for in silico analysis of promoter sequences. Nucleic Acids Research 2002, 30(1):325-327.

60. Krzywinski M, Schein J, Birol I, Connors J, Gascoyne R, Horsman D, Jones SJ, Marra MA: Circos: an information aesthetic for comparative genomics. Genome research 2009, 19(9):1639-1645.

61. Voorrips RE: MapChart: Software for the Graphical Presentation of Linkage Maps and QTLs. Journal of Heredity 2002, 93(1):77-78.

62. Kanehisa M, Araki M, Goto S, Hattori M, Hirakawa M, Itoh M, Katayama T, Kawashima S, Okuda S, Tokimatsu T et al: KEGG for linking genomes to life and the environment. Nucleic Acids Research 2008, 36(Database issue):D480-D484.

63. Mao X, Cai T, Olyarchuk JG, Wei L: Automated genome annotation and pathway identification using the KEGG Orthology (KO) as a controlled vocabulary. Bioinformatics 2005, 21(19):3787-3793.

64. Fernandez-Pozo N, Rosli HG, Martin GB, Mueller LA: The SGN VIGS tool: user-friendly software to design virus-induced gene silencing (VIGS) constructs for functional genomics. Molecular Plant 2015, 8(3):486-488.

65. Velasquez AC, Chakravarthy S, Martin GB: Virus-induced gene silencing (VIGS) in Nicotiana benthamiana and tomato. Journal of Visualized Experiments 2009(28): e1292.

\section{Figures}

\section{Figure 1}

Sequence alignment and phylogenetic analysis of the G2-like proteins of maize and tomato. (A) Phylogenetic tree of ZmGs and SIGlks. Each color represents one group, and five groups were found in total. (B) Alignment of the amino acid sequences of 66 putative G2-like genes in tomato. The different colors represent the different amino acids. 
A. Phylogenenic Tree

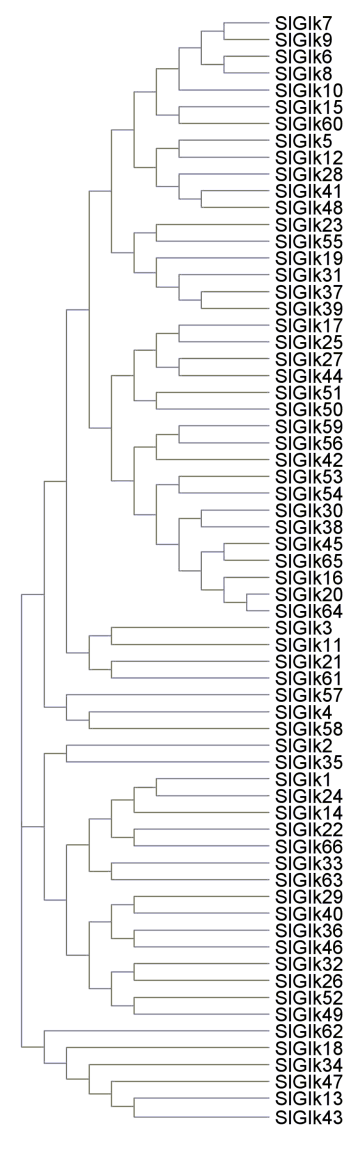

\section{B. Motif Patterns}

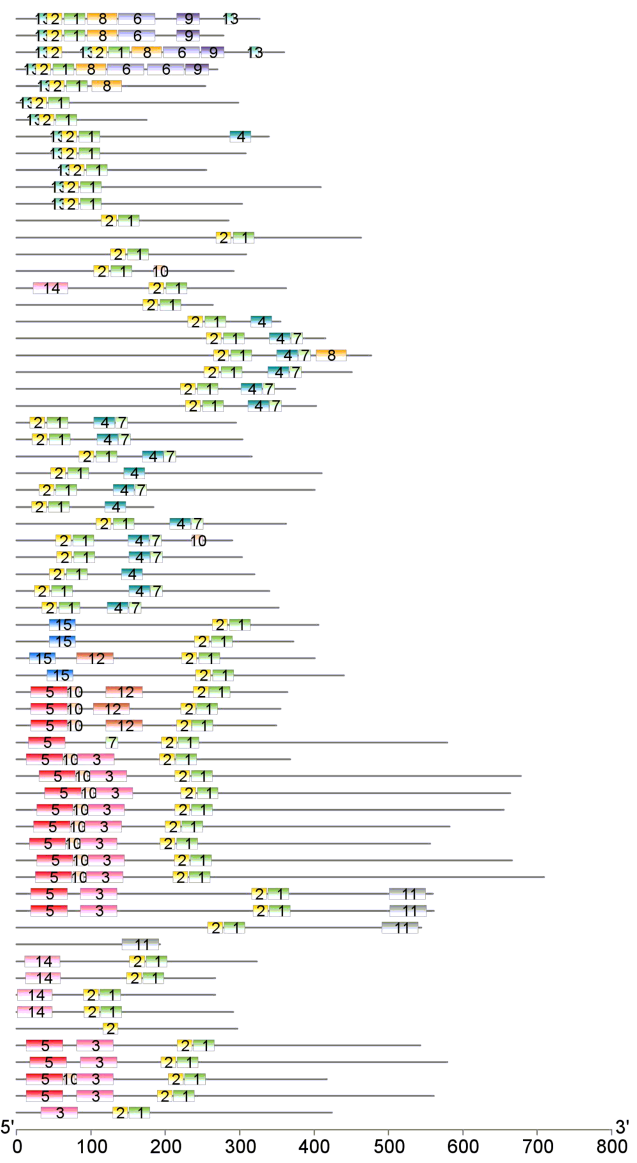

\section{Gene Structures}

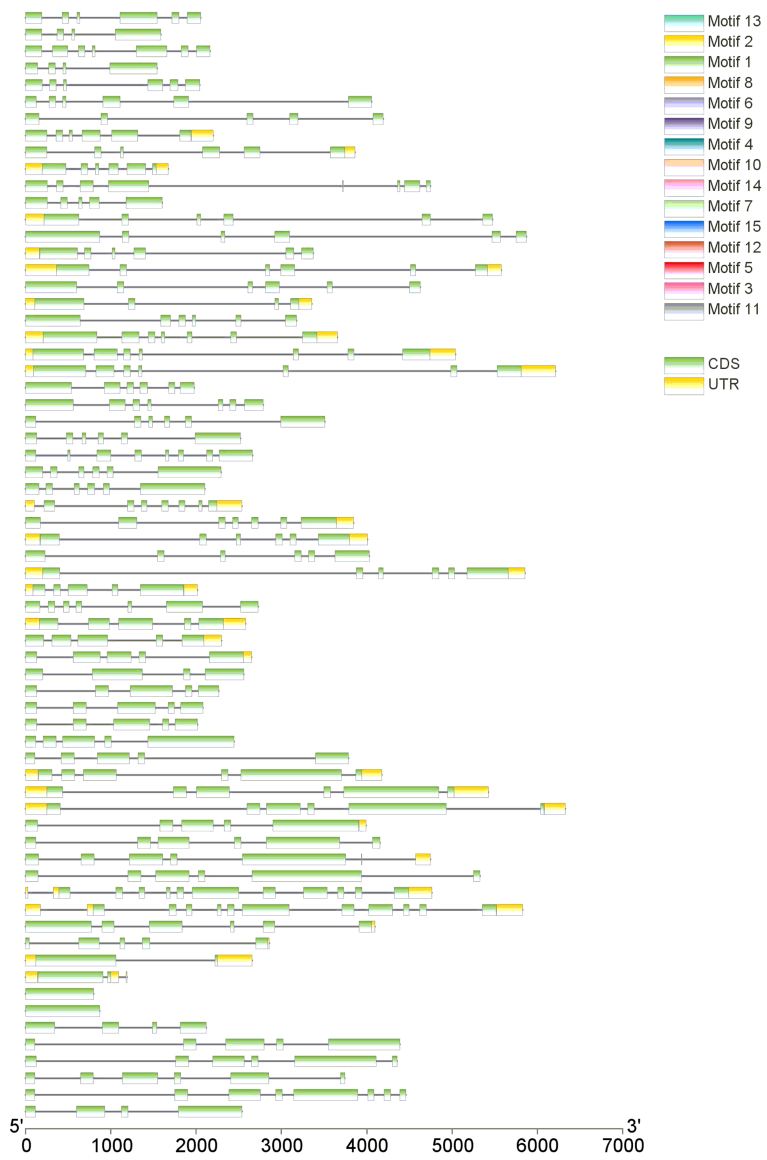

Figure 2

Phylogenetic tree, conserved protein motif structures and analysis of G2-like TFs-encoding genes from tomato. A. The phylogenetic tree was constructed by MEGA 7.0 software using G2-like protein sequences. B. Motif positions of tomato G2-like proteins. Fifteen motifs are displayed in boxes of different colors. C. Intron and exon analysis of 66 predicted tomato G2-like genes. The green and yellow boxes represent exon regions and untranslated regions (UTRs), respectively; black lines indicate introns. The scales at the bottom are shown for measuring the lengths of the motifs, exons and introns. 


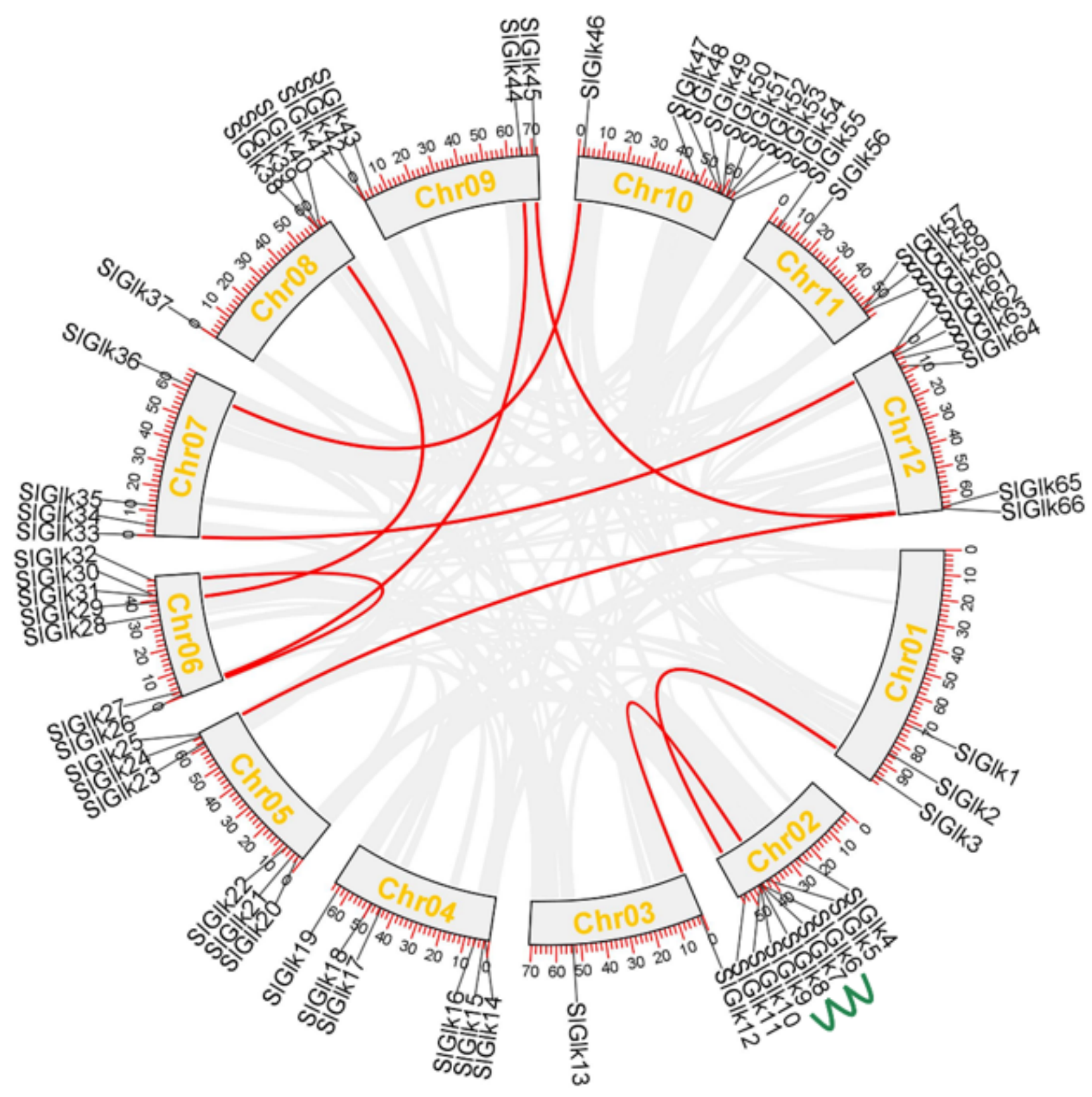

Figure 3

Chromosome distribution and duplication events of tomato G2-like genes. The different colored lines indicate different duplication events. The red lines indicate segmentally duplicated G2-like gene pairs, and the green lines indicate tandemly duplicated genes. The gray lines indicate all synteny blocks in the tomato genome. The lengths of the chromosomes can be estimated according to the scale provided. 


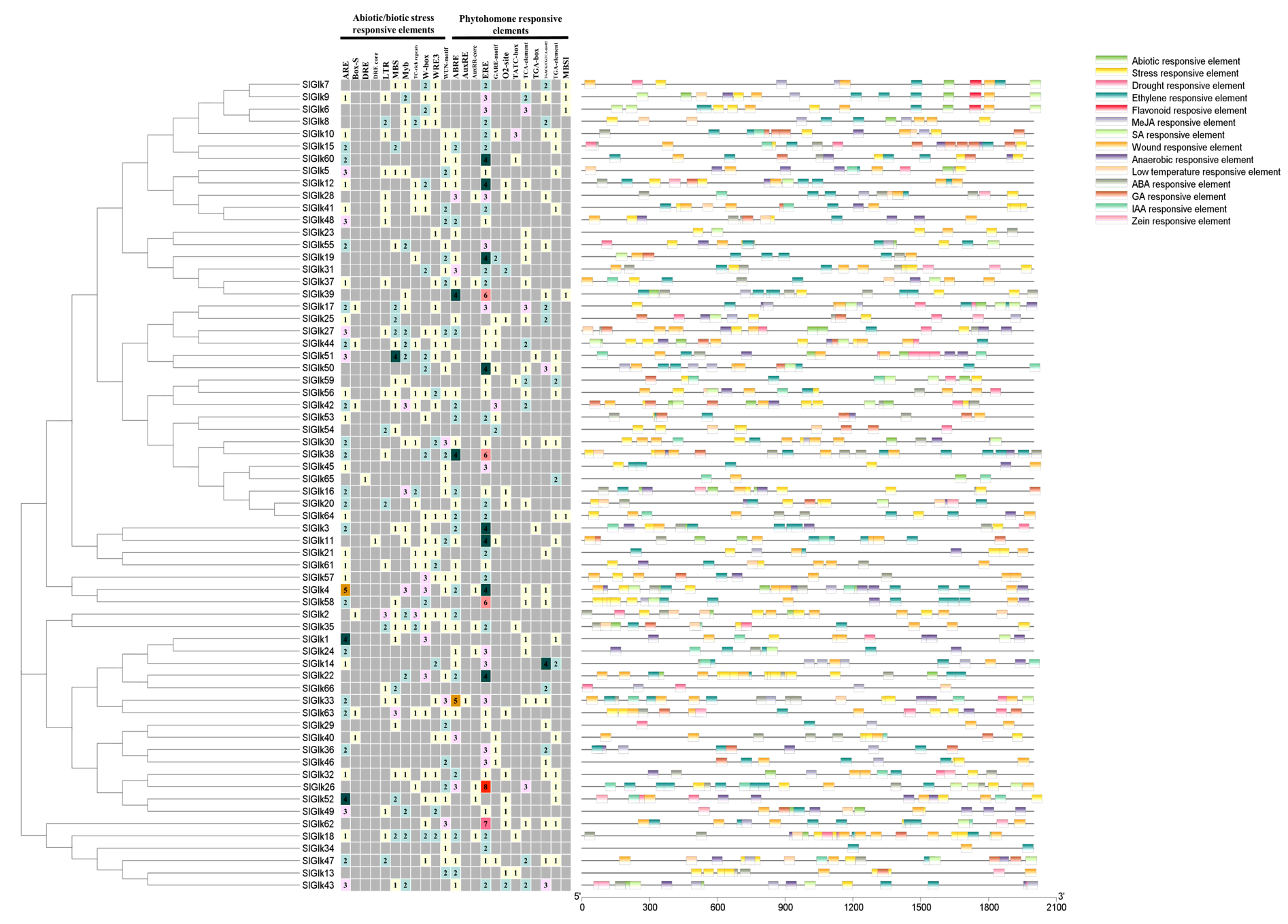

Figure 4

Analysis of CREs in promoter regions of SIGlks according to information in the Plantcare database. Left: numbers in different colors indicated the numbers of the main cis-acting elements of SIGIKs. Right: fifteen CREs were predicted, each of which is displayed in a different color. 


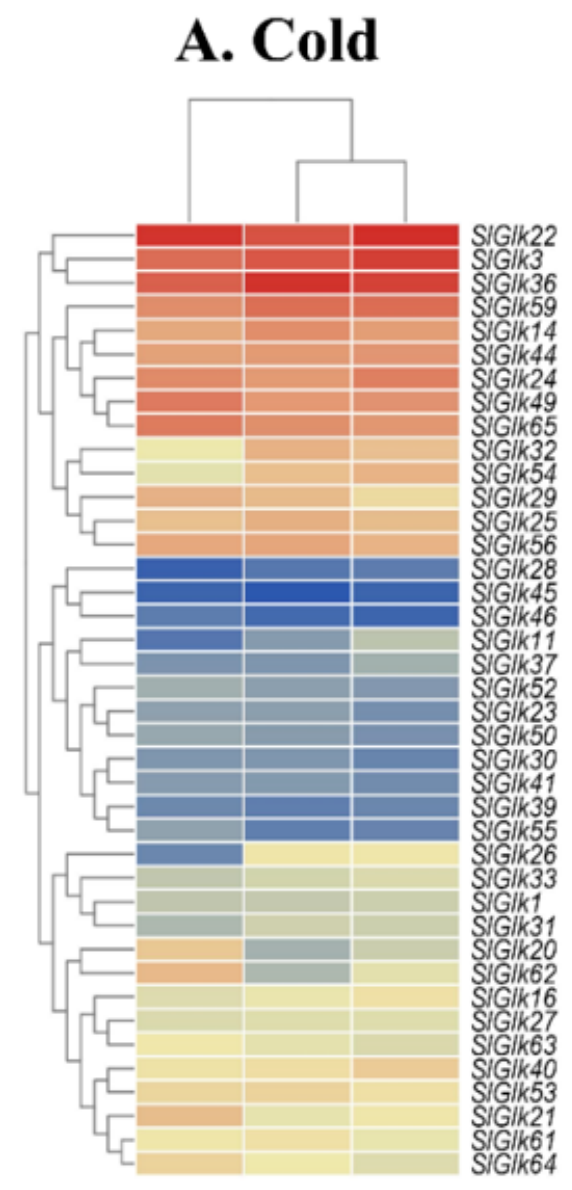

$0 \mathrm{~h} 4 \mathrm{~h} 12 \mathrm{~h}$
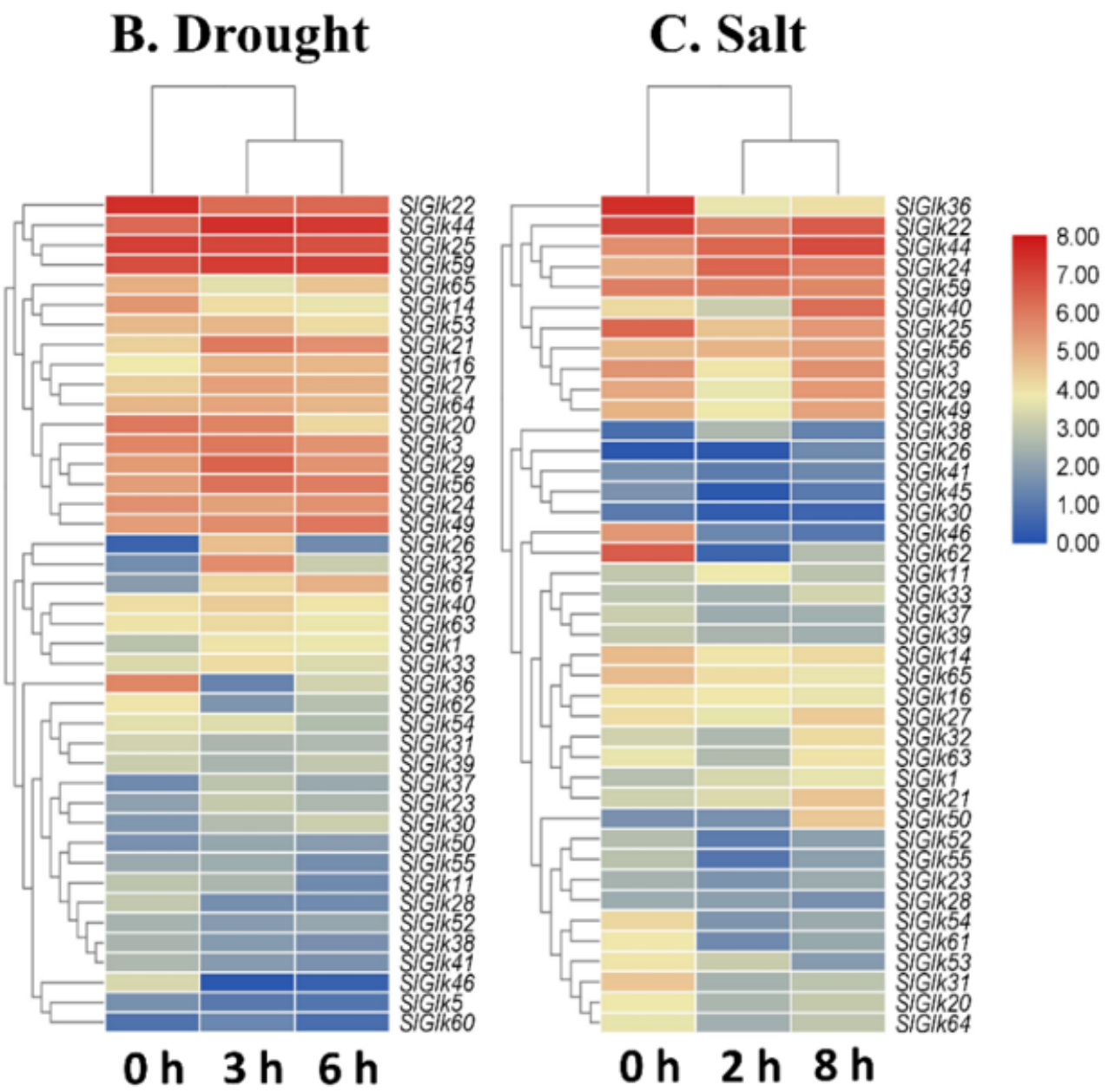

Figure 5

Expression profiles of SIGlk genes in response to three different abiotic stresses. Hierarchical clustering of the expression profiles of SIGlk genes in response to cold stress (A), drought stress (B) and salt stress (C). 
A

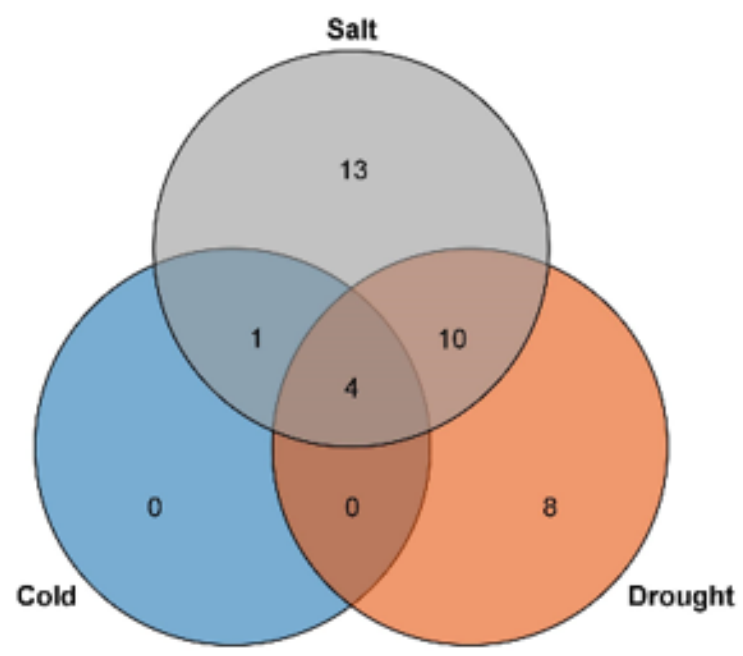

B

\begin{tabular}{|c|c|c|}
\hline Names & Total & Elements \\
\hline $\begin{array}{l}\text { Cold } \\
\text { Drought } \\
\text { Salt }\end{array}$ & 4 & $\begin{array}{ll}\text { SlGlk20 } & \text { SlGlk62 } \\
\text { SIGIk11 } & \text { SIGlk26 }\end{array}$ \\
\hline $\begin{array}{l}\text { Cold } \\
\text { Salt }\end{array}$ & 1 & SlGlk 54 \\
\hline $\begin{array}{l}\text { Drought } \\
\text { Salt }\end{array}$ & 10 & $\begin{array}{lll}\text { SIGlk30 } & \text { SIGIk61 } & \text { SIGlk46 } \\
\text { SIGIk22 } & \text { SIGlk44 } & \text { SIGIk21 } \\
\text { SIGIlk65 } & \text { SIGIk1 } & \text { SlGlk38 } \\
& \text { SIGIk36 } & \\
\end{array}$ \\
\hline Drought & 8 & $\begin{array}{ccc}\text { SIGlkAI } & \text { SIGlk23 } & \text { SlGlk32 } \\
\text { SIGlk28 } & \text { SlGlk16 } & \text { SlGlk37 } \\
\text { SIGlk14 } & \text { SlGlk5 }\end{array}$ \\
\hline Salt & 13 & $\begin{array}{ccc}\text { SIGlk31 SlGlk53 SlGlk52 } & \text { SlGlk50 } \\
\text { SIGlk34 SlGlk55 SlGlk3 } & \text { SlGlk64 } \\
\text { SIGlk40 SlGlk24 SlGlk45 } & \text { SIGlk25 } \\
\text { SIGlk29 } & \end{array}$ \\
\hline
\end{tabular}

音章

\begin{tabular}{|c|c|c|c|c|}
\hline SIGlk16 & $\mathrm{N}$ & u & $\mathrm{N}$ & \\
\hline SIGIK23 & $\mathrm{N}$ & u & $\mathrm{N}$ & \\
\hline SIGIk32 & $\mathrm{N}$ & u & $\mathrm{N}$ & \\
\hline SIGIK37 & $\mathrm{N}$ & u & $\mathrm{N}$ & Drought \\
\hline SIGIks & $\mathrm{N}$ & D & $\mathrm{N}$ & specific- related \\
\hline
\end{tabular}

SIGIkI4

SIGIk28

SIGIK4I

SIGIkII

Sigik3o

SiGik6l

SIGIk38

SIGIk3I

SIGIk53

SIGIk52

SIGIk55

siGIK3

SIGIk64

SIGIK45

SIGIk25

SIGIk29

SiGIk.50

SIGIk34

SiGikso

SiGlk24

SIGIK54

SiGiks4

SIGIk2I

SWGIKI

SiGik $\$ 6$

SiGik22

SIGIk65

SIGIk36

SIGIk20

SIGIK62

SIGIk26 specific- related

Salt

specific- related

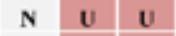

N U U

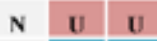

N D

N D D

Drought and salt related

\begin{tabular}{ll|l} 
N & D & D \\
\hline
\end{tabular}

\begin{tabular}{ll|l}
$N$ & $D$ & $D$ \\
\hline
\end{tabular}

D $\quad$ D 1

D D D

Common

Cold specific- related

\section{Figure 6}

Different response patterns of DEGs in response to three abiotic stress treatments. A. Venn diagram showing the overlap among genes differentially expressed in response to different abiotic stresses. B. Detailed list of DEGs in the Veen diagram. C. Representation of specifically expressed DEGs. U: Upregulated. D: Downregulated. N: No change. 


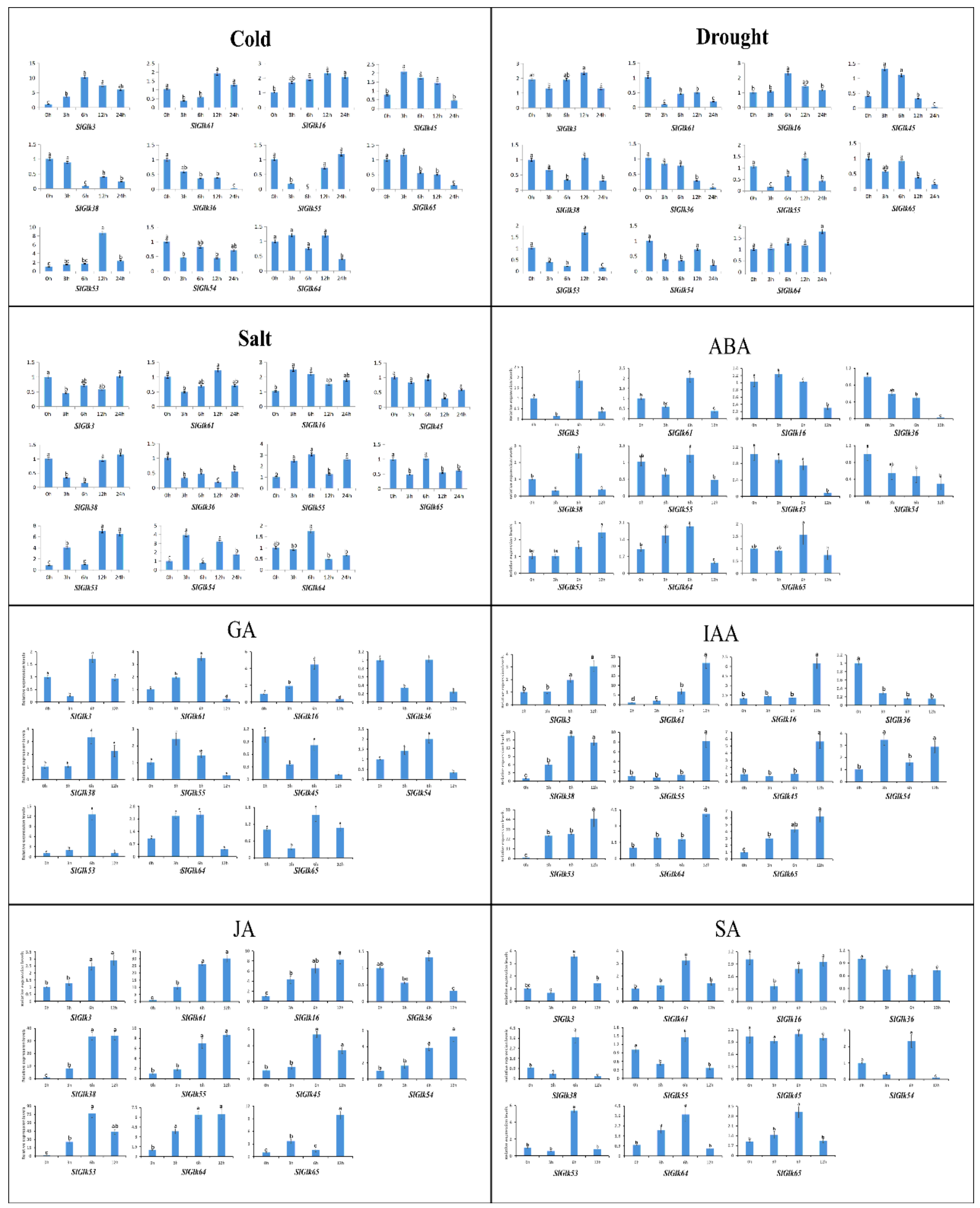

Figure 7

Expression patterns of SIGlks genes in response to abiotic stress and hormone treatments. The Y-axis indicates the relative expression level; the $X$-axis indicates hours of cold, salt, drought, $A B A, G A, I A A, J A$ and SA treatments. 
A

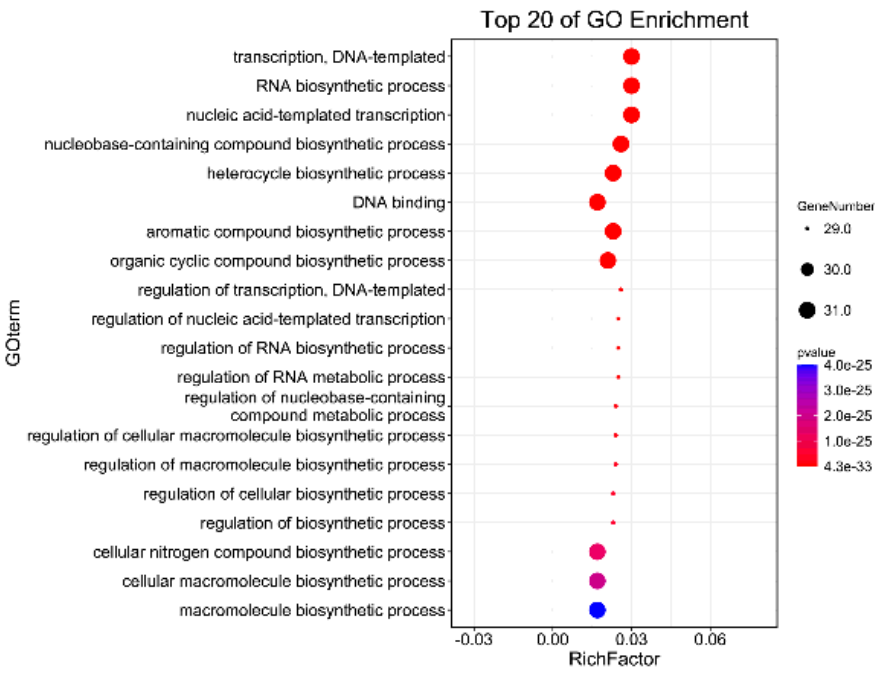

C

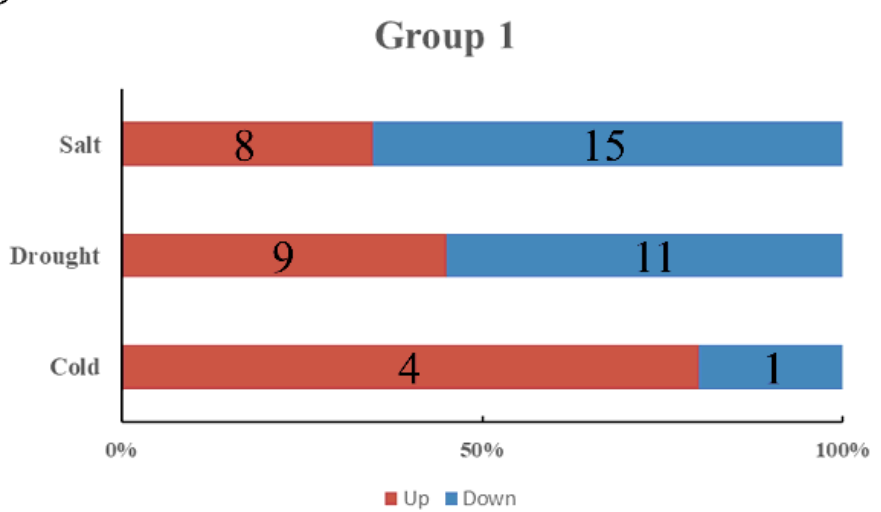

B

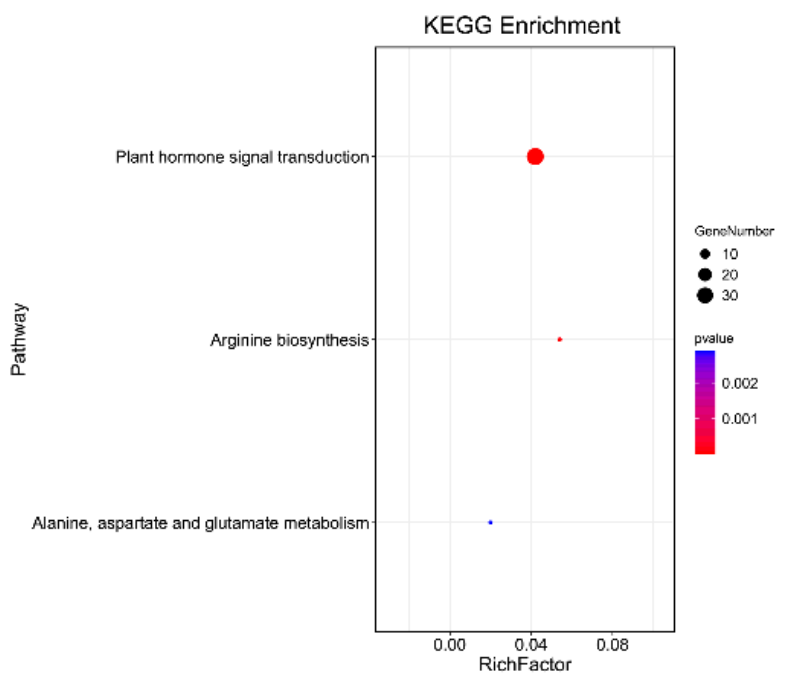

Group 2

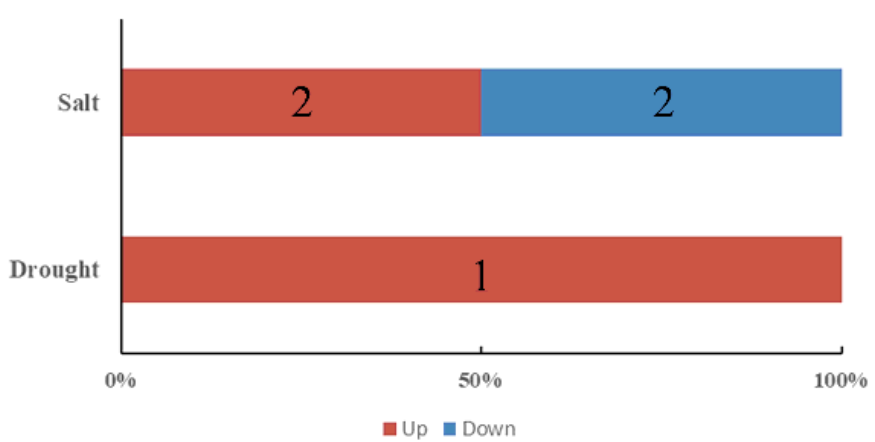

Figure 8

A. GO enrichment analysis of DEGs identified from RNA-seq. B. KEGG pathway enrichment of DEGs identified from RNA-seq. C. Comparisons of genes whose expression was upregulated and genes whose expression was downregulated between the two groups. 
A

$\mathbf{0 ~ h}$

$3 \mathbf{h}$

$6 \mathrm{~h}$

$12 \mathrm{~h}$
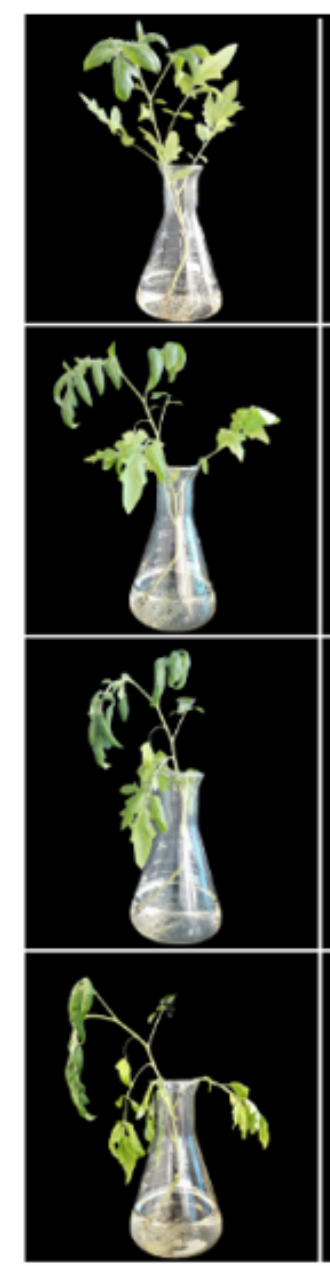

CK-TRV2 VIGS-SIGIk16
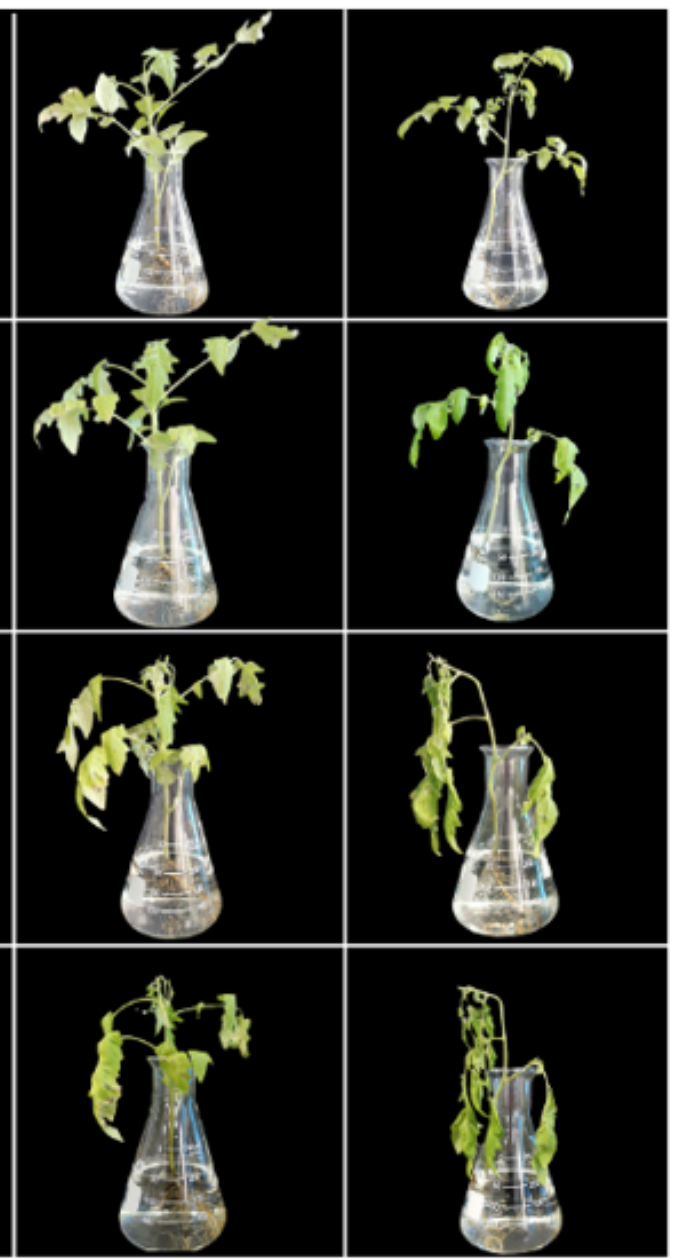
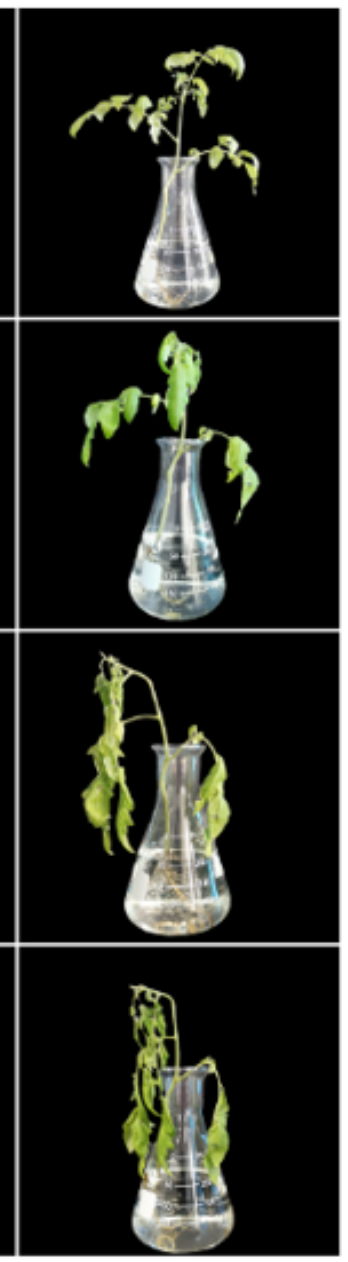

B

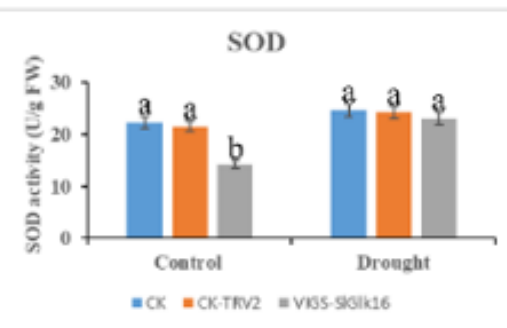

C

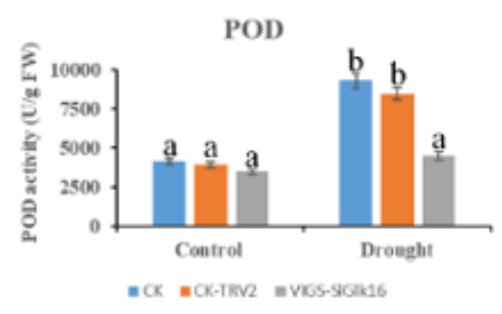

D

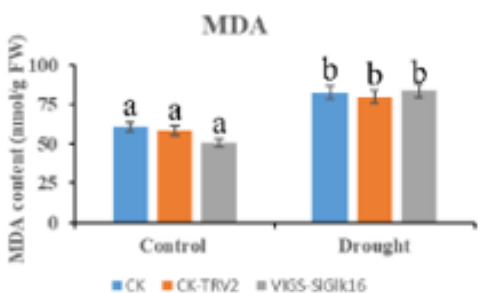

$\mathbf{E}$

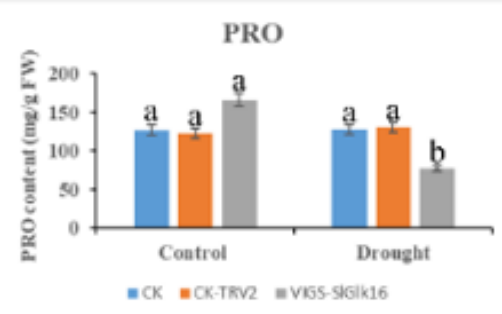

Figure 9

Silencing SIGlk16 reduced drought stress tolerance in tomato plants. A. Phenotypes of CK, CK-TRV2 and SIGIk16-silenced plants under drought stress. B. SOD and C. POD activities in CK, CK-TRV2 and SIGIk16silenced plants under normal and drought stress condition. D. MDA and E. Pro contents in CK, CK-TRV2 and SIGIk16-silenced plants under normal and drought stress condition.

\section{Supplementary Files}

This is a list of supplementary files associated with this preprint. Click to download.

- TestS1.txt

- TextS2.txt

- Fig1S.tif

- Fig.S2.tif

- FigS3.tif 
- 66.G2like.TableS1.xlsx

- 66.G2like.TableS2.xIsx

- 66.G2like.TableS3.xlsx

- 66.TableS4.docx

- 66.G2like.TableS5.xIsx

- 66.G2like.TableS6.xIsx

- 66.G2like.TableS7.xIsx

- 66.G2like.TableS8.xlsx

- 66.G2like.TableS9.xlsx 